\title{
Developing FoldGO, the tools for multifactorial functional enrichment analysis
}

\author{
A.M. Mukhin ${ }^{1,2 *}$, D.S. Wiebe ${ }^{1,2}$, I. Grosse ${ }^{2,3}$, S.A. Lashin ${ }^{1,2}$, V.V. Mironova ${ }^{1,2}$ \\ ${ }^{1}$ Institute of Cytology and Genetics SB RAS, Novosibirsk, Russia \\ ${ }^{2}$ Novosibirsk State University, Novosibirsk, Russia \\ ${ }^{3}$ Martin-Luther University, Halle-Wittenberg, Germany \\ *e-mail:mukhin@bionet.nsc.ru
}

Key words: functional enrichment analysis, differentially expressed genes, GO terms, web-service, R, RServe, Java, Spring, JavaScript, Vue.JS

Motivation and Aim: Due to development of whole-genome sequencing technologies researchers generate a big amount of tabular data about differentially expressed genes (DEGs), those with statistically significant change of transcription level in response to a stimulus or condition. Further analysis of DEGs is an actual task. DEGs are the genes, thats. One of the conventional methods of DEGs' analysis is Functional Enrtichment Analysis using Gene Ontology (GO) - a unified semantic system for gene annotation. In particular, this analysis provides for a list of enriched GO terms (molecular and biological function and cell component). There are online services DAVID and agriGO implemented this method. However, if one needs to study multiple connected gene lists, e. g., classified by the fold change, it is impossible to do it correctly in these services. The aim of this work was to develop a web-service implementing multifactorial functional enrichment analysis method.

Methods: We developed a new method, which classifies DEGs according to their fold change, performs functional annotation of the multiple gene lists generated on the basis of this classification (with overlaps) and provides for the GO terms, significantly overrepresented for the genes responded to the factor within a narrow fold-change-interval. The tools were developed using several programming languages and technologies:

- $\mathrm{R}$ - a programming language for R-package

- RServe - a library, which provides access to the R by using binary protocol

- Java - a programming language for writing a server, that will transmit $\mathrm{R}$ inputs and parameters

- Spring - a framework for developing web-services

- JavaScript - a programming language working in a browser for building a web-client

- Vue.JS - a library for building the interface

Results: The FoldGO tools are implemented as an $\mathrm{R}$ package and as a WebService. We tested FoldGO tools on a set of transcriptomes generated for different organisms, including Arabidopsis [1]. We found fold-change-specific GO categories within most of tested datasets. The web-service is deployed to the network of ICG SB RAS and the project is tested by users.

Acknowledgments: The work was supported RFBR grant No. 18-34-00871.

References

1. Omelyanchuk et al. (2017). 